\title{
A New Role for FGF2 as an Endogenous Inhibitor of Anxiety
}

\author{
Javier A. Perez, Sarah M. Clinton, Cortney A. Turner, Stanley J. Watson, and Huda Akil \\ Molecular and Behavioral Neuroscience Institute, University of Michigan, Ann Arbor, Michigan 48109
}

Human postmortem studies have demonstrated that fibroblast growth factor-2 (FGF2) expression is decreased in the brain of depressed individuals. It remained unclear, however, whether this is a consequence of the illness or whether FGF2 plays a primary role in the control of mood and emotions. In this series of studies, we first ask whether endogenous FGF2 expression correlates with spontaneous anxiety, a trait associated with vulnerability to severe mood disorders in humans. This is tested in two genetically distinct groups of rats selectively bred to differ dramatically in their response to novelty and anxiety-provoking conditions (HRs = low anxiety/high response to novelty vs LRs $=$ high anxiety/low response to novelty). We demonstrate that high-anxiety LRs have significantly lower levels of hippocampal FGF2 mRNA relative to low-anxiety HRs. We then demonstrate that FGF2 expression is modifiable by environmental factors that alter anxiety-thus, environmental complexity reduces anxiety behavior and induces FGF2 expression in hippocampus, particularly in highanxiety LRs. Finally, we directly test the role of FGF2 as an anxiolytic and show that a 3 week treatment regimen of peripherally administered FGF2 is highly effective at blunting anxiety behavior, specifically in high-anxiety LRs. This treatment is accompanied by an increase in survival of adult-born hippocampal cells, both neurons and astrocytes, most clearly in LRs. These findings implicate hippocampal FGF2 as a central integrator of genetic and environmental factors that modify anxiety, point to hippocampal neurogenesis and gliogenesis as key in this modulation, and underscore FGF2's potential as a new target for treatment of depression and anxiety disorders.

\section{Introduction}

Molecular factors imparting vulnerability or resilience to mood disorders remain elusive. Gene expression profiling in postmortem human brains has uncovered molecular changes that accompany these disorders. Our own studies have revealed that the fibroblast growth factor (FGF) system was the molecular ensemble most significantly altered in depressed individuals, across several cortical and subcortical regions (Evans et al., 2004; Akil et al., 2008). An independent study also reported under-expression of FGF2 in the hippocampus of depressed subjects (Gaughran et al., 2006), indicating a reliable association between depression and low FGFs.

It is difficult to distinguish whether these differences represent predisposing factors or are secondary to the disease process. Therefore, in the current series of animal studies, we ask how the FGF system relates to innate differences in emotional reactivity and whether manipulating it modulates emotionality. We focus on anxiety behavior, given the significant comorbidity between clinical anxiety and depression and evidence that these disorders share a common genetic etiology (Gorwood, 2004). We focus on FGF2 because, of 22 FGF family members, it is the best studied in brain and shows consistent changes in depression. FGF2 interacts with four receptors (Ornitz, 2000; Reuss and von Bohlen und Halbach, 2003), all of which are expressed in brain (Eckenstein,

Received 0ct. 7, 2008; revised March 16, 2009; accepted April 2, 2009

This research was supported by National Institute of Mental Health Grant R36 MH078694, National Institute on Drug Abuse Grant 5 P01 DA021633, Office of Naval Research Grant N00014-02-1-0879, and The Pritzker Neuropsychiatric Disorders Research Fund L.L.C. We are grateful to Jennifer Fitzpatrick, Tracy Simmons, James Stewart, and James Beals for their technical support throughout the completion of these studies.

Correspondence should be addressed to Javier A. Perez, Molecular and Behavioral Neuroscience Institute, University of Michigan, 205 Zina Pitcher Place, Ann Arbor, MI 48109. E-mail: perezja@umich.edu.

DOI:10.1523/JNEUROSCI.4829-08.2009

Copyright $\odot 2009$ Society for Neuroscience $\quad$ 0270-6474/09/296379-09\$15.00/0
1994; Belluardo et al., 1997). It is critical for brain development (Raballo et al., 2000) and modulates hippocampal neurogenesis in the developing (Wagner et al., 1999) and adult brain (Palmer et al., 1995; Pieper et al., 2005).

We rely, here, on an animal model wherein Sprague Dawley rats were genetically selected to enhance differences in emotional behavior (Stead et al., 2006). After several rounds of breeding, the two lines of high responders (HR) versus low responders (LR) exhibit dramatic differences in exploration of a novel environment. Importantly, they display consistent differences in several tests of spontaneous anxiety, with HRs exhibiting low and LRs exhibiting high-anxiety behavior. We underscore that these lines are more distinct in their behavioral and neural phenotypes than is observed in outbred HR and LR rats. Thus, beyond showing the expected differences in drug-seeking behavior (Davis et al., 2008), the bred lines exhibit significant differences in several measures of emotionality (Stead et al., 2006; Flagel et al., 2008). The novelty response in these lines is highly heritable and stable: it is fully predictable based on family background, develops early in life, is not dramatically altered when pups are cross-fostered by dams from the opposing line, and is not affected by prenatal stress (Clinton et al., 2008). In contrast, their anxiety behavior, although highly heritable, is to some extent influenced by maternal care. Thus, they offer an excellent model of genetic differences in "emotional reactivity," including inborn differences in anxiety that can be altered by experience.

We also manipulate the environment by increasing the complexity of the rats' housing conditions and asking whether this differentially alters FGF2 and impacts anxiety in the selectively bred lines, testing for gene by environment interactions. Finally, we administer exogenous FGF2 to directly ascertain its role in 
anxiety behavior in the HR-LR lines and characterize its impact on proliferation and survival of adult-born hippocampal cells.

\section{Materials and Methods}

Animals. Adult Male Sprague Dawley rats were obtained from our inhouse breeding colony at the Molecular and Behavioral Neuroscience Institute where we have maintained the selectively bred HR-LR lines for over 19 generations. HR-LR lines were selectively bred based on their differences in exploratory response to novelty, a characteristic initially used to predict individual differences in drug-taking behavior (Piazza et al., 1989). We recently published a description of our breeding strategy and an initial behavioral characterization of the HR-LR differences in anxiety-like behavior (Stead et al., 2006). We have also shown that HR-LR differences in novelty response is likely genetic, as it shows little change when animals are cross-fostered to a mother from the opposing line. Anxiety responses, although highly predictable based on genetic background, are somewhat modifiable by maternal conditions (Clinton et al., 2007). Adult rats (300-400 g) $\sim 2$ months old were housed two animals per cage; (one HR and one LR were housed together as a pair) after locomotor screening under a $12 \mathrm{~h}$ light/dark cycle (lights on at 6:00 A.M.) with food and water available ad libitum. Animals were allowed to acclimate to the housing conditions for at least $7 \mathrm{~d}$ before any experiments. Behavioral experiments were run in two separate cohorts with all groups represented tested in parallel. The presented data are the sum of the two different cohorts run. The neurogenesis study is part of the last cohort of animals that was used for the FGF2 behavioral experiments. Each in situ hybridization study had its own cohorts in which no behavioral experiments were performed. All animals were treated in accordance with the National Institutes of Health Guidelines on Laboratory Animal Use and Care and in accordance with the guidelines set by the university committee on use and care of animals at the University of Michigan.

Locomotion testing. At postnatal day 55-60, adult male rats from our selective breeding colony were screened for locomotor response to a novel environment to confirm the HR-LR phenotype by placing them in a standard size $(43 \times 21.5 \times 24.5)$ clear acrylic cage in a different room from where the animals had been housed. Locomotor activity was monitored every $5 \mathrm{~min}$ for $1 \mathrm{~h}$ by two panels of photocells connected to a computer. The first panel of three photocells was placed at ground level to record horizontal locomotion, with the second panel of five photocells located near the top of the cage to determine rearing behavior. The locomotion-testing rig and motion-recording software were created inhouse at the University of Michigan. Locomotion activity was tested between 9:00 and 11:30 A.M. Final locomotion scores were determined by summing horizontal and rearing activities.

Light-dark box anxiety test. The light-dark box (LDB) apparatus is a $30 \times 60 \times 30 \mathrm{~cm}$ Plexiglas shuttle box with a translucent cover. The floor is composed of stainless steel bars suspended above corncob bedding. Each box is divided into two equal-sized compartments by a wall with a $12-\mathrm{cm}$-wide open door. One compartment is painted white and brightly illuminated, and the other is painted black with very dim light. Time spent in each compartment is monitored by rows of five photocells located $2.5 \mathrm{~cm}$ above the grid floor of each compartment, and total time spent in each compartment is recorded with a microprocessor. Animals who spend less time in the illuminated compartment and more time in the dark compartment are classified as showing greater anxiety behavior.

Elevated plus maze. The elevated plus maze (EPM) is constructed of black Plexiglas, with four elevated arms $(70 \mathrm{~cm}$ from the floor, $45 \mathrm{~cm}$ long, and $12 \mathrm{~cm}$ wide). The arms are arranged in a cross, with two opposite arms enclosed by $45-\mathrm{cm}$-high walls, and the other two arms open. At the intersection of the open and closed arms, there is a central $12 \times 12 \mathrm{~cm}$ square platform giving access to all arms. The test room is dimly lit $(\sim 40$ lux), and behavior is monitored using a computerized video tracking system (Noldus Ethovision). At the beginning of the 5 min test, each rat is placed in the central square facing a closed arm. The computerized tracking system records the latency to first enter the open arm, the amount of time spent in the open arm, closed arm, and center square over the course of the 5 min test. Behavior testing is performed between 8:00 and 11:30 A.M.
Environmental complexity. To increase the complexity of the environment, HR-LR rats were housed for $21 \mathrm{~d}$ in $3 \times 3 \times 3$ feet stainless steel cages which contain different toys, obstacle courses, and enriching stimuli. The number of toys is increased over the course of the 3 weeks of environmental complexity (EC). Thus, every day, animals are exposed to novel sensory stimuli and increased opportunities for exploration as part of their housing environment. During the EC experiment, control HR and LR animals were handled once every other day, matching the time duration of handling of the EC animals. This was done to account for the handling necessitated when introducing additional toys for the EC group.

Chronic FGF2. The chronic FGF2 regimen involves the administration of either FGF2 (5 ng/g, i.p) or vehicle ( 0.1 м PBS with $0.1 \%$ BSA) every day for 3 weeks. Systemic injections with this dose have previously been shown to alter neurogenesis (Wagner et al., 1999). The 3 week treatment period is used to match the duration of the EC housing period. Animals are then tested for anxiety measures starting $1 \mathrm{~d}$ after the last injection.

$m R N A$ in situ hybridization. At the conclusion of each experiment, rats were killed by rapid decapitation and their brains removed, snap frozen in isopentane, and stored at $-80^{\circ} \mathrm{C}$. Brains were cryostat sectioned at $-20^{\circ} \mathrm{C}$ at $20 \mu \mathrm{m}$ for the EC studies or $10 \mu \mathrm{m}$ for the basal HR-LR studies and sliced in series throughout the hippocampus, mounted on Super Frost Plus slides (Fisher Scientific), and stored at $-80^{\circ} \mathrm{C}$ until processed. In situ hybridization methodology has been previously described in detail elsewhere (Kabbaj et al., 2000). The FGF2 probe was labeled in a reaction mixture consisting of $1 \mu \mathrm{g}$ of linearized plasmid, $1 \times$ transcription buffer (Epicenter Technologies), $125 \mu \mathrm{Ci}$ of ${ }^{35} \mathrm{~S}$-labeled UTP, $125 \mu \mathrm{Ci}$ of ${ }^{35} \mathrm{~S}$ CTP, $150 \mu \mathrm{M}$ ATP and GTP, $12.5 \mathrm{~mm}$ dithiothreitol, $1 \mu \mathrm{l}$ of RNase inhibitor, and $1.5 \mu \mathrm{l}$ of T7 RNA polymerase. Radioactive signals were quantified using computer-assisted optical densitometry software (Scion Image Beta 4.03; Scion Corporation). Integrated densities were determined by outlining the region of interest from both hemispheres. Optical density measurements were corrected for background, and the signal threshold defined as the mean gray value of background plus $3.5 \times$ its SD. Only pixels with gray values exceeding the above-defined threshold are included in the analysis. In the present studies, optical density measurements were taken for four subregions of the hippocampus (hippocampus fields CA1-CA3 and the dentate gyrus) from the left and right sides of the brain. Data from multiple sections per animal were averaged resulting in a mean integrated optical density value for each animal and then averaged for each group. On average, a total of 12 sections per animal (10-14 sections per brain) were used for the analysis of in situ hybridization. Section sampling spanned from $-2.3 \mathrm{~mm}$ from bregma through -5.6 $\mathrm{mm}$, and animals were matched in rostral to caudal comparison according to Paxinos and Watson (1998).

Bromodeoxyuridine/Ki67 immunohistochemistry. To assess the effect of FGF2 on hippocampal new cell survival, rats were injected with bromodeoxyuridine (BrdU; Calbiochem) at a dose of $200 \mathrm{mg} / \mathrm{kg}$ dissolved in saline once daily for $2 \mathrm{~d}$ before the start of the FGF2 treatment paradigm. Twenty-four hours after BrdU labeling, animals underwent $21 \mathrm{~d}$ of FGF2 treatment before being exposed to behavior testing after which they were killed by decapitation and brain was snap frozen. For determining the rate of cell proliferation, we performed immunohistochemical labeling of Ki-67, which is an endogenous marker of ongoing cell proliferation. For BrdU and Ki67 immunohistochemistry, a series of every eight sections is cut throughout the entire extent of the hippocampus at $30 \mu \mathrm{m}$ and slide mounted. For Ki-67 DAB staining, sections are postfixed in $4 \%$ paraformaldehyde for $1 \mathrm{~h}$, followed by a $45 \mathrm{~min}$ incubation in $10 \mathrm{~mm}$ sodium citrate at $90^{\circ} \mathrm{C}$. Sections are then rinsed with PBS and washed in $0.3 \%$ peroxide followed by blocking with BSA containing $1 \%$ goat serum and $0.05 \%$ Triton X-100. Subsequently, sections are incubated overnight with rabbit polyclonal anti-ki67 (University of Michigan) 1:40000 in BSA. After PBS washes, sections are incubated in biotinylated goat antirabbit secondary antibody 1:1000 (Vector laboratories) followed by avidin/biotin complex (Vectastain Elite $\mathrm{ABC}$ kit) and subsequent $\mathrm{DAB}$ reaction for visualization of signal. For DAB staining of $\mathrm{BrdU}$, sections are postfixed in $4 \%$ paraformaldehyde for $1 \mathrm{~h}$, rinsed in PBS, and washed in $0.3 \%$ peroxide. Sections are then incubated in $50 \%$ formamide $-2 \times$ SSC at $65^{\circ} \mathrm{C}$ for $2 \mathrm{~h}$ followed by two $5 \mathrm{~min}$ rinses in $2 \times$ SSC. Slides are then placed for $30 \mathrm{~min}$ in $2 \mathrm{~N} \mathrm{HCl}$ at $37^{\circ} \mathrm{C}$ and $10 \mathrm{~min}$ in $0.1 \mathrm{M}$ boric acid at 
room temperature, followed by rinsing in PBS and blocked with BSA containing $1 \%$ goat serum and $0.05 \%$ Triton X-100. Sections are incubated overnight at room temperature with rat monoclonal anti-BrdU (Accurate) 1:1000 in BSA. After PBS washes, sections are then incubated in biotinylated goat anti-rat (Vector Laboratories) secondary antibody 1:1000 followed by avidin/biotin complex amplification (Vectastain Elite ABC kit) and subsequent $\mathrm{DAB}$ reaction for visualization of signal. Cresyl violet staining is performed for both immunostains, and sections are subsequently dehydrated through graded alcohols followed by immersion in xylene and then coverslipped with Permount mounting medium. For fluorescent triple labeling, we use 1:1000 dilution of rat anti-BrdU in combination with 1:1000 dilution of mouse monoclonal anti-GFAP (Millipore Bioscience Research Reagents). For fluorescent secondary antibodies, we use Alexa 594 goat antirat and Alexa 647 goat anti-mouse (Invitrogen). For NeuN labeling, we use a primary monoclonal mouse anti-NeuN antibody tagged with an Alexa 488 Fluor (Millipore Bioscience Research Reagents).

Cell counting. For quantification of DAB-stained Ki-67 and BrdU cells, slides are initially coded, and the code is not broken until counts are analyzed to ensure that a blind observer performs all cell counts. To estimate the total number of cells, we follow a modified unbiased stereological procedure used by Malberg and Duman (2003), whereby the total number of cells counted per animal is multiplied by the reciprocal of the sampling factor. BrdU and Ki67 cells are counted on a light microscope under $63 \times$ objective in the granule cell layer and subgranular zone (SGZ) of the hippocampus located at the border of the granule cell layer. Cells are included in SGZ counts if the cell is near the SGZ or touching the SGZ and excluded if the cell is more than two cell diameters from the SGZ. In triple labeling experiments, at least $30 \mathrm{BrdU}$ cells are examined per subject to determine the percentage of BrdU-positive cells that colabel with NeuN or GFAP using a laser-scanning confocal microscope. Laser scans of $0.5 \mu \mathrm{m}$ serial $Z$-section planes are visualized using a $63 \times$ objective to determine the neuronal or glial differentiation of BrdU-positive cells. To obtain an estimate of the total number of new neurons and new glial cells in the hippocampus of each animal, we use the percentage of BrdU + cells colabeled with NeuN, GFAP, or neither and multiplied it by the total number of BrdU+ cells for each animal.

Statistical analyses. Behavioral studies and anatomical studies were analyzed by ANOVAs followed by Fisher's least statistical discrimination post hoc comparisons. All data are presented as mean \pm SEM, and statistical significance is assumed at $p<0.05$.

\section{Results}

FGF2 expression in the hippocampus is lower in selectively bred high-anxiety LR animals relative to low-anxiety HR animals

To elucidate the role of FGF2 in modulating anxiety behavior, we examined basal levels of FGF2 gene expression in the selectively bred HR and LR animals, which have been previously shown to display basal differences in anxiety behavior. We specifically began examining the hippocampus, as this region has been previously shown to be the primary target region modulating HR-LR differences in anxiety-like behavior (Kabbaj et al., 2000) and is a region where FGF2 shows prominent neuronal expression and exhibits dysregulation in human depressed subjects. As seen in Figure $1 A$, illustrated in Figure $1 B$, the high-anxiety LR animals have significantly lower basal levels of FGF2 expression in the dentate gyrus $\left(F_{(1,16)}=6.63, p<0.05\right)$ and CA3 $\left(F_{(1,16)}=4.5\right.$, $p<0.05)$ relative to the less anxious HR animals.

\section{Environmental complexity differentially reduces anxiety-like} behavior in the selectively bred high-anxiety LR animals

Since EC has been previously shown to influence emotionality by reducing anxiety (Benaroya-Milshtein et al., 2004), we asked whether this manipulation would have differential effects in the HR versus LR animals, given their basal differences in anxiety behavior. This behavioral study was also a prelude to assessing the impact
A.

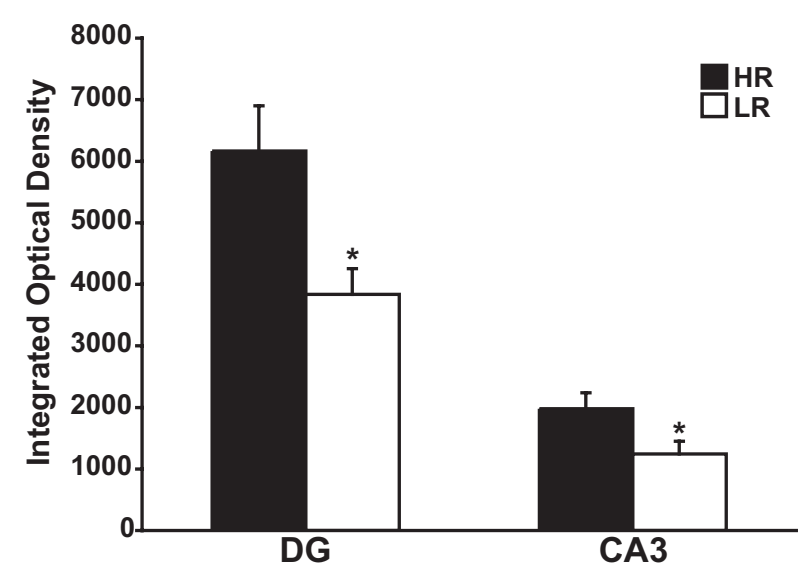

\section{B.}

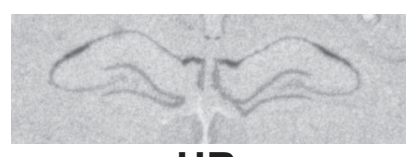

HR

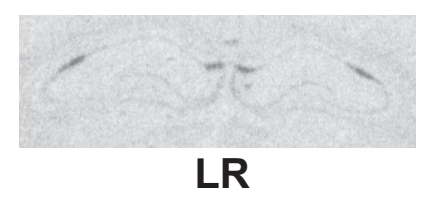

Figure 1. Hippocampal FGF2 gene expression in selectively bred HR-LR animals. $\boldsymbol{A}$, Relative to HRs, LR animals show significantly lower basal levels of FGF2 gene expression in the dentate gyrus and CA3 region of the hippocampus. Integrated optical density data are presented as mean \pm SEM. ${ }^{*} p<0.05$ from ANOVA comparisons of HR $(n=10)$ and $\operatorname{LR}(n=8)$ animals. $\boldsymbol{B}$, Representative section images from $H R$ and $L R$ animals showing significantly lower basal levels of FGF2 gene expression in the hippocampus of LRs relative to HRs.

of this environmental manipulation on FGF2 expression in the two lines of rats. To determine the effects of EC on anxiety behavior in the bred HR and LR animals, we used the LDB and EPM tests, as both of these measures have previously been used to characterize the behavioral differences between these selectively bred lines.

In the EPM, as expected, there is a significant main effect of phenotype, whereby HR animals display lower anxiety behavior compared with LR animals as measured by the percentage of open arm entries $\left(F_{(1,52)}=8.1, p<0.01\right)$ (Fig. $\left.2 A\right)$. A significant main effect is also seen in response to $\mathrm{EC}$, where EC increased the percentage of open arm entries $\left(F_{(1,52)}=20.0, p<0.0001\right)$ indicating the anxiolytic impact of this manipulation on both groups.

In the LDB, there is also significant main effect of EC on anxiety-like behavior, as evidenced by an overall increase in the percentage of time spent in the illuminated compartment $\left(F_{(1,35)}\right.$ $=6.02, p<0.05$ ) by the EC-housed relative to standard-housed animals (Fig. $2 B$ ). Interestingly, although $\mathrm{HR}$ animals spend an overall higher percentage of their time in the illuminated compartment relative to LRs as demonstrated by a main effect of HR-LR phenotype $\left(F_{(1,35)}=7.7, p<0.01\right)$, this is primarily attributable to the basal differences (i.e., under control, non-EC conditions) and not the EC condition. This is confirmed by the finding of a significant interaction effect between HR-LR phenotype and treatment (control vs EC) $\left(F_{(1,35)}=6.8, p<0.05\right)$. Post hoc tests reveal a differential effect of EC on LR, with LR-EC animals displaying significantly less anxiety behavior than LR control animals $(p<0.01)$. Thus, the EC manipulation abolishes the differences in spontaneous anxiety between HR and LR animals, showing that in the LDB test, EC has a selective impact in alleviating the anxiety behavior of the high-anxiety LRs. 
A.

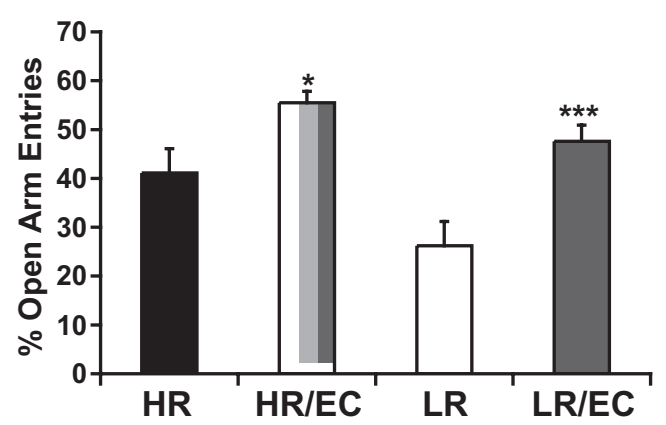

B.

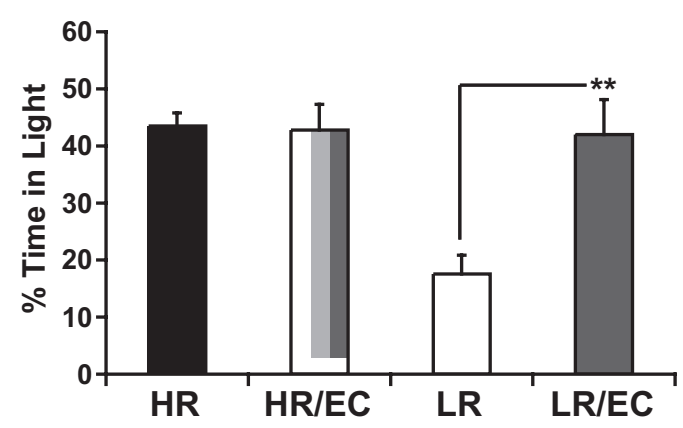

Figure 2. Effects of EC on anxiety-like behavior in HR and LR animals in the EPM $(\boldsymbol{A})$ and LDB (B). Under standard housing conditions, $L R$ animals display higher anxiety behavior relative to $H R s$; however, exposure to $\mathrm{EC}$ makes $\mathrm{LR}$ anxiety behavior resemble that of an HR animal. $A$, In the EPM, both $H R$ and $L R$ animals benefit from a $21 \mathrm{~d}$ exposure to $E C$ with a decrease in anxiety as shown by an increase in the percentage of open arm entries; ${ }^{*} p<0.05$, comparison between $H R$ and HR-EC; ${ }^{* * *} p<0.001$ comparison between LR and LR-EC. $B$, However, in the LDB, EC exposure for $21 \mathrm{~d}$ significantly decreases anxiety in $\mathrm{LR}$ animals only as shown by a significant increase in the percentage of time spent in the illuminated compartment; ${ }^{* *} p<0.01$. Data are presented as mean \pm SEM from ANOVA comparisons with Fisher's post hoc test comparisons ( $n=13-15$ per group) resulting from the sum of two independent cohorts containing all experimental groups.

\section{Environmental complexity differentially increases FGF2} expression in the hippocampus of selectively bred highanxiety $L R$ animals

Our results indicate that $\mathrm{HR}$ animals have higher basal levels of FGF2 expression in the hippocampus and display lower anxiety relative to LR animals. Given the finding that EC reduced anxiety behavior more consistently in the LR animals, we asked whether EC resulted in increased FGF2 expression in the hippocampus and whether this effect was differential across the two selectively bred lines of rats.

The results in Figure 3 show a main effect of housing condition, wherein EC leads to an overall significant increase in FGF2 expression in the dentate gyrus $\left(F_{(1,19)}=23.3, p<0.001\right)$ and CA3 region $\left(F_{(1,19)}=16.7, p=0.001\right)$. Moreover, there is a significant interaction between EC housing and HR-LR phenotype on FGF2 expression in the dentate gyrus $\left(F_{(1,19)}=6.17, p<0.05\right)$ and in the CA3 region $\left(F_{(1,19)}=6.4, p<0.05\right)$. Post hoc analysis reveals that LR-EC animals exhibit higher levels of FGF2 in the dentate gyrus $(p<0.0001)$ and CA3 region $(p<0.001)$ compared with LR controls, showing an impact of EC on gene expression in these animals. In contrast, EC did not exert any effects on FGF2 expression in the bred HRs. Thus, the high-anxiety selectively bred LRs show a greater impact of EC on both their anxiety behavior and FGF2 expression in the hippocampus, leading us to hypothesize that FGF2 plays a role in mediating the decrease in anxiety that results from exposure to a complex environment.
It should be noted that in this specific experiment, basal differences in FGF2 expression are lost in the CA3 between HRs and LRs controls. However, it is also worth noting that experimental conditions varied considerably between this and the previous experiment. As stated in Materials and Methods, in this experiment, control HRs and LRs were handled every other day, whereas in the previous experiment, animals were left undisturbed until killed. We believe that handling of LR animals might have resulted in an increase in FGF2 expression in the CA3, which might have contributed to a loss in differences in FGF2 expression in the CA3 between HRs and LRs. This notion is not far fetched, since handling can decrease anxiety, which as we suggest is associated with changes in FGF2 expression. However, a direct experimental comparison between handled and unhandled animals would be required to show this conclusively.

\section{Chronic FGF2 differentially reduces anxiety-like behavior in} the selectively bred high-anxiety LR animals

Given the results above showing that both genetic endowment and environmental conditions modulate hippocampal FGF2 expression, with higher levels of this growth factor being correlated with reduced anxiety behavior, we set out to directly test the hypothesis that FGF2 is a key regulator of anxiety behavior. We, therefore, administered FGF2 chronically and asked whether it would alter measures of anxiety behavior in both the highanxiety LRs and the low-anxiety HRs in the LDB and EPM.

As illustrated in Figure 4, our results show the expected difference in the HR-LR phenotype in the LDB test. Importantly, they reveal an overall anxiolytic effect of FGF2 treatment. Thus, as seen in Figure $4 A$, HR animals overall spend more time in the illuminated compartment of the $\operatorname{LDB}\left(F_{(1,46)}=38.5, p<0.0001\right)$. Moreover, FGF2 treatment results in an overall increase in the percentage of time spent in the illuminated compartment of the LDB, $\left(F_{(1,46)}=5.9, p<0.05\right)$. More importantly, there is an interaction effect of treatment with phenotype $\left(F_{(1,46)}=5.6, p<\right.$ 0.05). Post hoc analysis reveals FGF2 treatment having a significant anxiolytic effect in FGF2-treated LRs relative to vehicletreated LRs $(p<0.01)$, whereas no significant effect of FGF2 treatment is observed in the HR rats.

A similar pattern is seen in the EPM. A significant interaction effect of chronic FGF2 treatment $\times$ phenotype is observed on measures of anxiety behavior in the EPM, including percentage of open arm entries $\left(F_{(1,46)}=7.2, p<0.01\right)$ (Fig. $\left.4 B\right)$ and percentage of time spent in the open $\operatorname{arm}\left(F_{(1,46)}=6.1, p<0.05\right)$ (Fig. $4 C)$. Further post hoc analysis reveals that LR animals differentially benefit from the anxiolytic effects of chronic FGF2 treatment, whereas HR animals do not. Thus, FGF2-treated LRs show a significant increase in the percentage of open arm entries $(p<$ $0.01)$ and in the percentage of time spent in open arms $(p<0.01)$ relative to vehicle-treated LRs. No significant effects are seen when comparing these measures in FGF2-treated versus vehicletreated HRs.

\section{FGF2 differentially increases new cell survival in the selectively bred high-anxiety LRs}

Given that EC is well known for increasing neurogenesis and that it produced a significant increase in FGF2 gene expression in the dentate gyrus, it was reasonable to hypothesize that increase in neurogenesis after EC may be mediated at least in part by FGF2. Indeed, exogenous treatment with FGF2 has been shown to increase neurogenesis in the dentate gyrus of the hippocampus (Wagner et al., 1999; Pieper et al., 2005). Our specific question was whether the treatment protocol that we used, chronic periph- 
A.

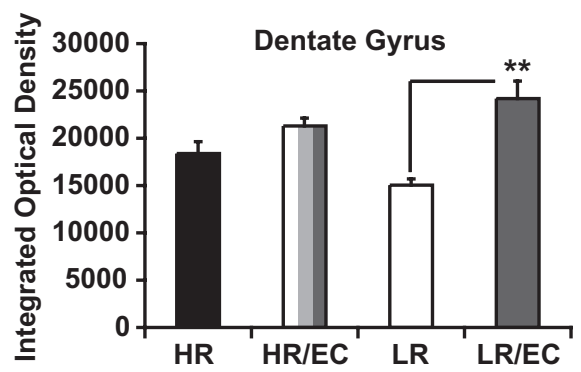

B.

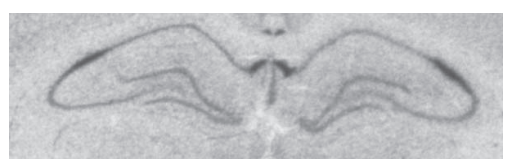

HR

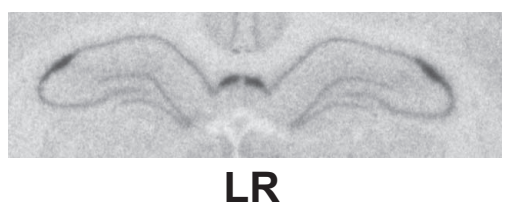

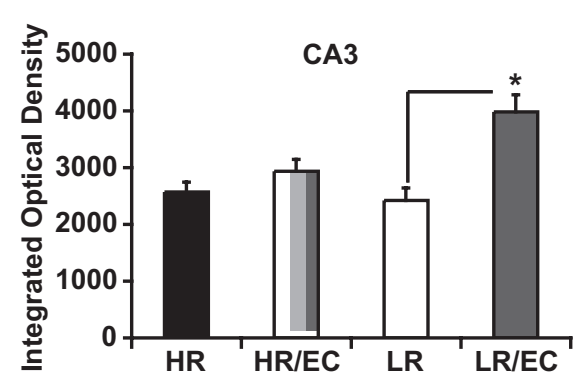

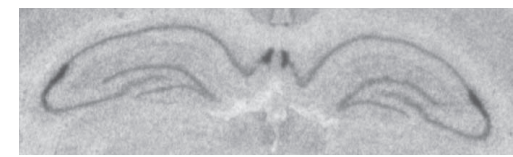

HR/EC

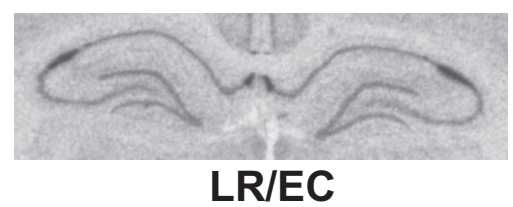

a $19 \%$ increase). In contrast, FGF2 produces a substantial increase in the total number of BrdU-labeled cells in the LR rats (up to $3802 \pm 286$ ), representing an $85 \%$ increase. Thus, there is a significant interaction of treatment $\times$ phenotype $\left(F_{(1,16)}=5.2, p<0.05\right)$. Similar findings, showing a differential increase in LRs are observed when counting the total number of BrdU cells in the hilus region (data not shown).

It is noteworthy that these results appear to be independent of an increase in cell proliferation, as we observed no basal differences in proliferation at the time of death between HRs and LRs and as chronic FGF2 failed to increase the number of Ki67-labeled cells $\left(F_{(1,16)}=1.1 p>0.05\right)$ (Fig. $5 B$ ). No changes in proliferation are observed when specifically counting the hilus region either (data not shown).

The combination of data from the Ki67 and BrdU markers suggests that HR and LR lines tend to have some basal differences in the turnover of adult-born cells in the hippocampus, with the more anxiety-prone LRs showing similar birth but lower rates of survival. Remarkably, FGF2 treatment appears to increase cell survival only in the LR animals, in conjunction with their more consistent decrease in anxiety behavior.

FGF2 differentially alters adult-born cell differentiation in the hippocampus of selectively bred HR versus LR animals Given the differential increase in cell survival in LRs in response to FGF2, we evaluated the pattern of differentiation of the surviving BrdU-labeled cells and obtained an estimate of the total number of neurons and astrocytes generated in the hippocampal dentate gyrus as a function of treatment with FGF2.

As depicted in Figure $6 A$, there are basal differences in the pattern of differentiation of adult-born hippocampal cells between selectively bred HR and LR rats, with the bred HRs having a significantly higher percentage of differentiated astro-

eral FGF2 administration, would alter hippocampal neurogenesis and if it would do so in a manner that correlates with the alterations in anxiety behavior-i.e., more markedly in the LR rats that are more responsive to the treatment.

As shown in Figure 5, there is no significant main effect of HR-LR phenotype; however, HR and LR animals show a nonsignificant tendency to exhibit basal differences in number of BrdUlabeled cells $(2739 \pm 295$ in HR and $2061 \pm 127$ in LR) $(p=$ $0.08)$. In contrast, there is a significant main effect of treatment as chronic administration of FGF2 results in a significant increase in new cell survival in the dentate gyrus as shown by an increase in the number of BrdU-labeled cells $\left(F_{(1,16)}=18.5, p<0.001\right)$ (Fig. $5 A)$. Interestingly, chronic administration of FGF2 produces little change in the total number of BrdU cells in HRs (3270 \pm 306 , cytes relative to LRs $\left(F_{(1,16)}=5.77, p<0.05\right)$. However, bred LRs exhibit a significantly greater percentage of undifferentiated BrdU-labeled cells, termed "neither" $\left(F_{(1,16)}=7.73, p<0.05\right)$. Furthermore, as seen in Figure 6A, FGF2 treatment alters the percentage of undifferentiated cells $\left(F_{(1,16)}=9.1, p<0.05\right)$ and shows a significant interaction with phenotype $\left(F_{(1,16)}=26.9\right.$, $p<0.001)$. Post hoc tests show a highly significant decrease in the percentage of undifferentiated cells selectively in the bred LRs $(p<0.0001)$. Thus, chronic treatment with FGF2 alters the percentage of "uncommitted cells" in the bred LRs while eliminating basal differences between HRs and LRs in the percentage of GFAP-labeled cells.

Figure $6 B$ captures the resulting distribution of each cell type across phenotype and treatment conditions, and the resulting 
A.

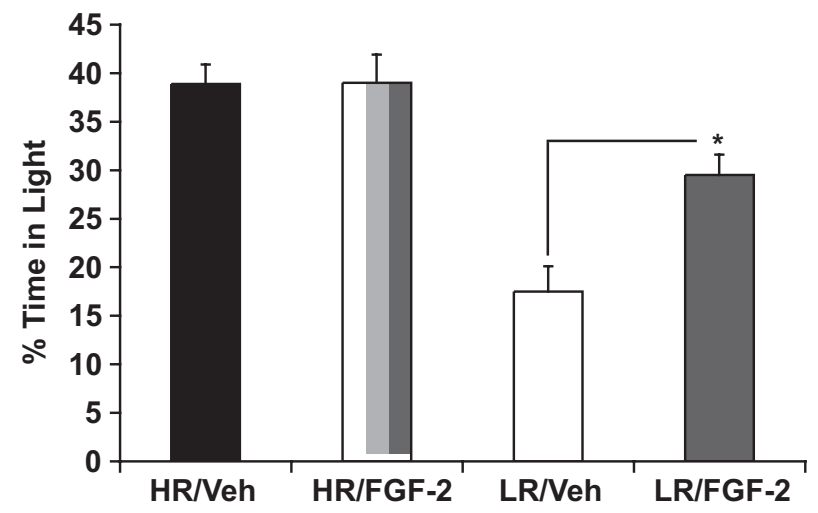

B.

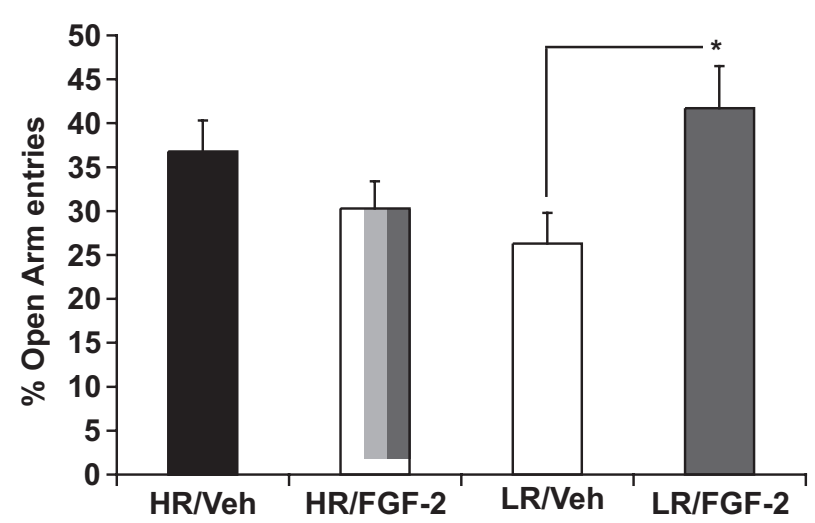

C.

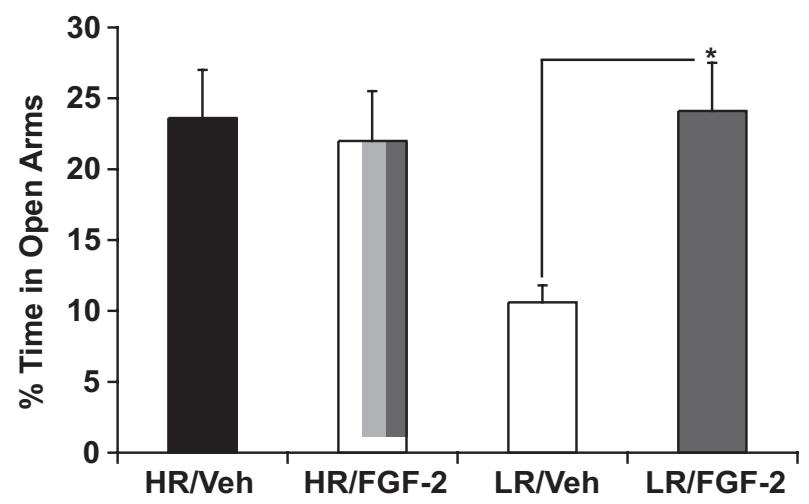

Figure 4. Effects of chronic FGF2 treatment on anxiety-like behavior in HR and LR animals LDB $(\boldsymbol{A})$ and EPM $(\boldsymbol{B}, \boldsymbol{C})$. $\boldsymbol{A}$, Twenty-one days of chronic FGF2 treatment significantly reduced anxiety-like behavior selectively in $L R$ animals as seen by an increase in the time spent in the illuminated compartment of the LDB. B, C, Similarly, chronic treatment with FGF2 showed selective anxiolytic effects in LRs on the EPM as seen by an increase in the percentage of open arm entries $(\boldsymbol{B})$ and in the percentage of time in open arms $(\boldsymbol{C})$ with no effects observed in HRs on either of the measures. Data are presented as mean \pm SEM from ANOVA comparisons with Fisher's post hoc test comparisons. ${ }^{*} p<0.01$ ( $n=11-15$ per group) resulting from the sum of two independent cohorts in which all experimental groups were represented.

absolute numbers are summarized in Table 1. Statistical analyses show several main effects as well as interactions between HR-LR phenotype and the impact of FGF2 treatment on the various cell populations. Beyond the tendency for the basal differences in total number of BrdU-labeled cells described above, HR and LR animals exhibit basal differences in the number of cells where BrdU is colabeled with GFAP or neither (Table 1, Fig. $6 B$ ). In
A.

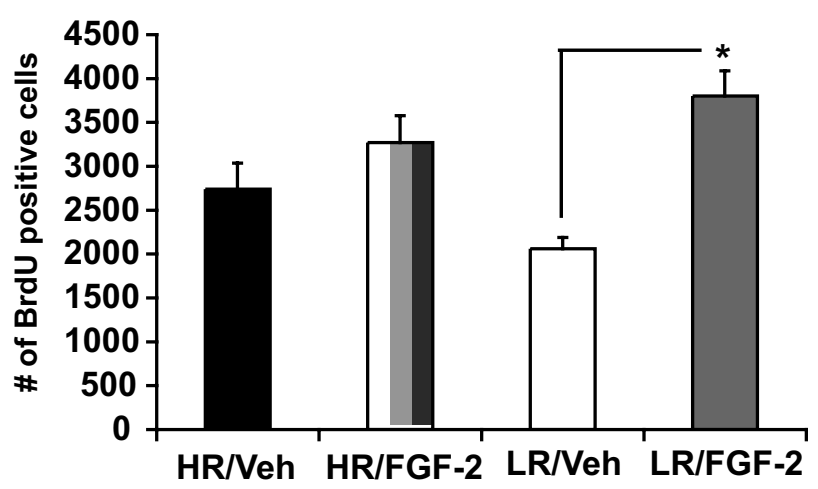

B.

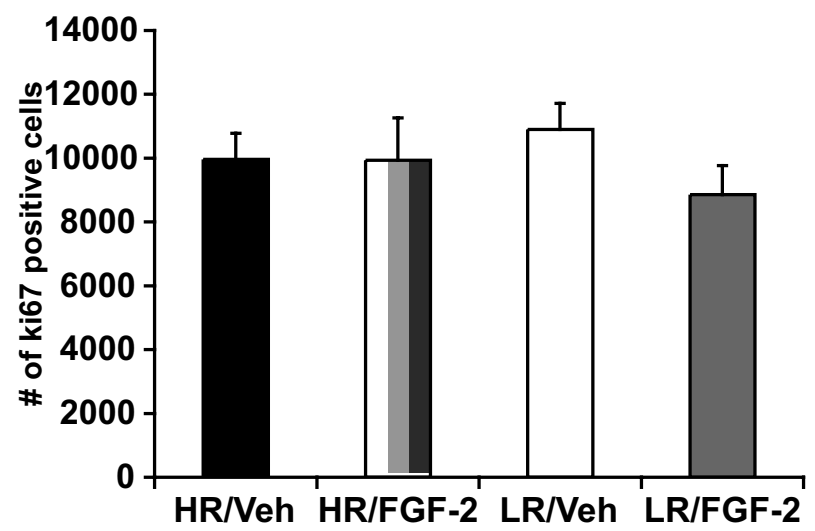

Figure 5. Effects of chronic FGF2 treatment on hippocampal cell genesis in HR and LR animals. To assess the effect of FGF2 on hippocampal new cell survival, rats were injected with BrdU at a dose of $200 \mathrm{mg} / \mathrm{kg}$ once daily for $2 \mathrm{~d}$ before the start of FGF2 treatment. Twenty-four hours after BrdU labeling, animals underwent $21 \mathrm{~d}$ of FGF2 treatment. $\boldsymbol{A}$, Twenty-one days of chronic FGF2 treatment significantly increases the survival of adult-born cells in the hippocampus of LRs as shown by an increase in the number of BrdU-labeled cells, whereas no change is seen in HRs. $\boldsymbol{B}$, These effects on hippocampal cell genesis were independent of changes in cell proliferation as chronic FGF2 treatment failed to show any significant impact in the number of Ki67-labeled proliferating cells. Data are presented as mean \pm SEM from ANOVA comparisons with Fisher's post hoc test comparisons. ${ }^{*} p<0.001$ ( $n=5$ per group).

particular, relative to LRs, HRs show a higher number of new astrocytes generated in the dentate gyrus $\left(F_{(1,16)}=27.1, p<\right.$ $0.0001)(235 \pm 25$ in HR vs $62 \pm 4$ in LR), and as shown by post hoc analysis, this is particularly attributable to the difference in the control groups $(p<0.0001)$. An interaction effect was also observed on the number of undifferentiated cells $\left(F_{(1,16)}=9.5\right.$, $p<0.01$ ), with post hoc tests revealing that under vehicle conditions LRs show a higher number of undifferentiated cells $(p<$ $0.01)$ relative to vehicle HRs. FGF2 treatment resulted in an overall increase in the number of new astrocytes $\left(F_{(1,16)}=33.1, p<\right.$ $0.0001)$ and new neurons $\left(F_{(1,16)}=20.2, p<0.001\right)$ as a main effect, but the impact is different as a function of HR-LR phenotype. Thus, there were significant interaction effects in the number of newly generated neurons $\left(F_{(1,16)}=9.7, p<0.01\right)$, astrocytes $\left(F_{(1,16)}=9.6, p<0.01\right)$, as well as undifferentiated cells $\left(F_{(1,16)}=9.5, p<0.01\right)$.

As seen in Figure $6 B$ and Table 1 , in the HR animals, FGF2 produces small increases in all cell groups ranging from $13 \%$ for neurons, 23\% for astrocytes, and 52\% $(p<0.01)$ for neither marker cells. In contrast, in the LR animals, FGF2 produces a 
A.

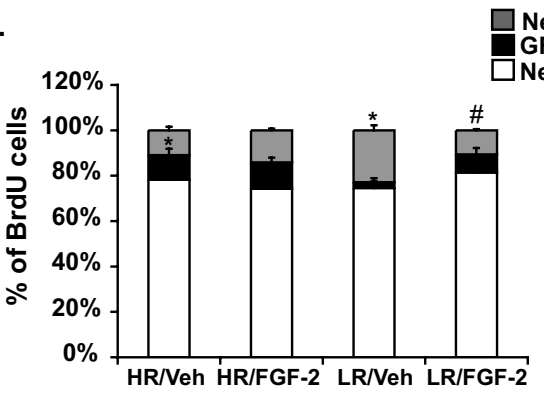

B.

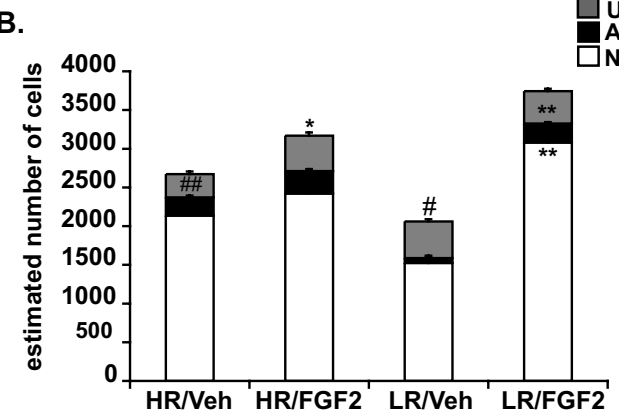

C.
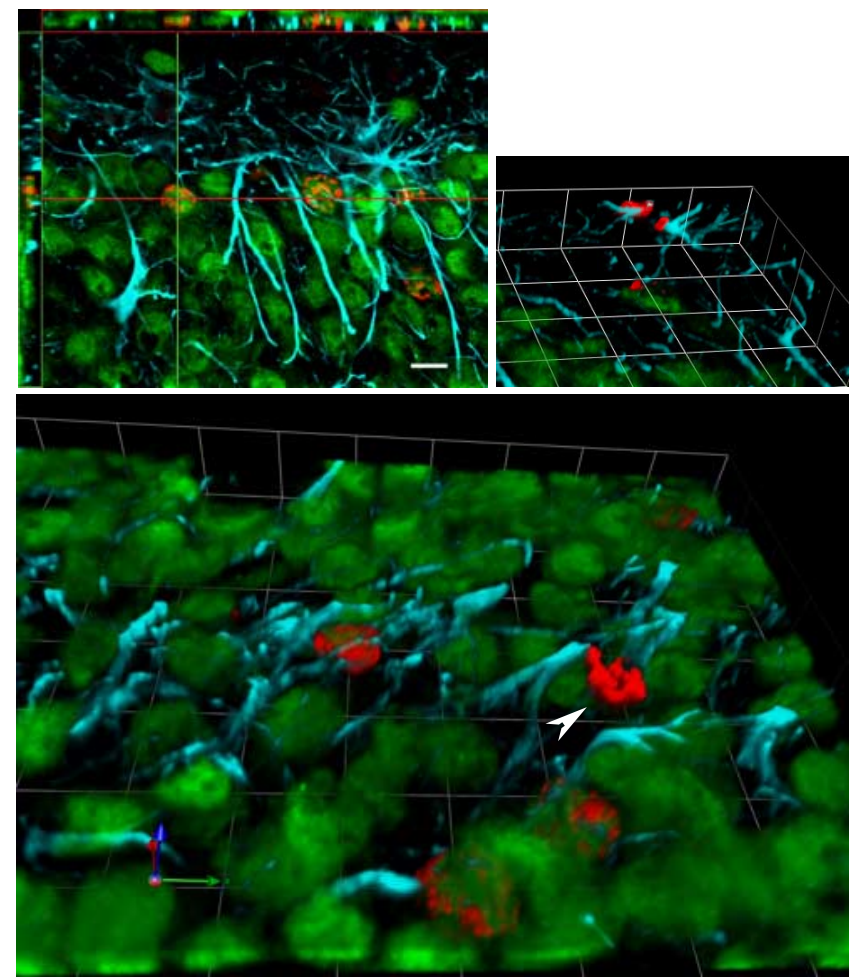

Figure 6. Effects of chronic FGF2 treatment on cell differentiation of adult-born cells in the hippocampus of HR and LR animals. $A$, Under vehicle conditions, HRs show a greater percentage of astrocyte differentiation relative to LRs, whereas $L R$ animals show a greater percentage of undifferentiated cells. Chronic treatment with FGF2 disrupts such differences in cell differentiation between both phenotypic groups as FGF2 treatment selectively decreases the percentage of undifferentiated cells in LRs. ${ }^{*} p<0.05,{ }^{\#} p<0.0001$. $B$, Under vehicle conditions, HRs show a higher proportion of newly generated astrocytes (BrdU +, GFAP + cells) relative to LRs, which show a higher population of undetermined cells (BrdU +, GFAP-, NeuN-). Differential effects of FGF2 on BrdU cell survival in LRs significantly increases the proportion of newly generated neurons (BrdU +, NeuN + cells) and astrocytes (BrdU +, GFAP + cells). ${ }^{\#} p<0.01,{ }^{\# \#} p<$ $0.0001,{ }^{*} p<0.01,{ }^{* *} p<0.0001$. Data are presented as mean \pm SEM from ANOVA comparisons with Fisher's post hoc test comparisons ( $n=5$ per group). $C$, Representative confocal images of BrdU/NeuN- and BrdU/GFAP-colabeled cells and an undifferentiated BrdU-labeled cell; red labeling are BrdU-positive cells, blue labeling are GFAP-positive cells, and green are NeuN-positive cells. Top left, A BrdU-labeled cell colabeled with NeuN confirmed in the substantial increase in BrdU-labeled cells, and Figure $6 \mathrm{~B}$ and Table 1 show that this represented a doubling of neurons $(201 \%$ increase over basal; $p<0.0001)$ and a remarkable fourfold increase in astrocytes (398\% over basal; $p<0.0001)$. These results show that FGF2 treatment eliminates HR and LR differences in the number of newly generated astrocytes in the hippocampus.

\section{Discussion}

The present study establishes for the first time a central role for hippocampal FGF2 in modulating anxiety behavior. Our findings are as follows: (1) Selectively bred HR rats, who exhibit lower spontaneous anxiety behavior, have higher basal levels of hippocampal FGF2 mRNA relative to their LR counterparts. (2) Enriching the environment decreases anxiety behavior and leads to increased expression of FGF2 mRNA. The more anxious LRs profit more from this treatment, showing increased FGF2 expression and decreased anxiety. (3) Chronic treatment with exogenous FGF2 decreases anxiety behavior, and this is seen only in LRs. (4) Chronic treatment with exogenous FGF2 increases new cell survival in hippocampus without any detectable changes in proliferation. This leads to an overall increase in neurogenesis and particularly glial genesis. Once again, this effect is seen primarily in the bred LRs. These results strongly implicate hippocampal FGF2 as an inhibitor of anxiety behavior and suggest that it produces this behavioral effect by altering hippocampal circuitry. Although the impact of basal FGF2 on anxiety derives from genetic endowment, environmental factors such as enrichment can compensate for genetic vulnerability, especially benefiting individuals prone to high anxiety.

Several strands of evidence demonstrate the relevance of FGFs to emotional behavior. First, we and others have found that FGF family members, including FGF2, are decreased in postmortem brains of severely depressed individuals (Evans et al., 2004; Gaughran et al., 2006; Akil et al., 2008). Moreover, FGF2 decreases in social defeat, an animal model of depression (Turner et al., 2006), and FGF2 and related molecules function as antidepressants in the Porsolt Swim Test (Turner et al., 2008b). Finally, chronic treatment with classical antidepressants increases FGF2 expression (Mallei et al., 2002) and enhances neurogenesis (Malberg et al., 2000). This body of work is consonant with the neurotrophic hypothesis that a deficiency in growth factors imparts increased vulnerability to depression (Duman and Monteggia, $2006,2008 \mathrm{a}$ ), that induction of these factors is key to the effectiveness of antidepressants (Warner-Schmidt and Duman, 2007), and that enhanced neurogenesis is required for antidepressant action (Santarelli et al., 2003). Multiple neurotrophic factors likely contribute to the beneficial actions of antidepressants (Martinowich et al., 2007; Warner-Schmidt and Duman, 2007).

It remained unclear, however, whether FGF2 only responds to negative environmental manipulations (e.g., social stress, the illness itself) or whether it contributes to intrinsic vulnerability to anxiety and depression. This study demonstrates that FGF2 is a genetic predisposing factor, a mediator of environmental influences, and a key player in the interaction of genes with the environment in determining the anxiety phenotype. As importantly, it shows that the impact of FGF2 is not only seen under patho-

\section{$\leftarrow$}

$x, y / x, z$ axis (scale bar, $10 \mu \mathrm{m}$ ). Top right, Three-dimensional image of a pair BrdU cells colabeled with GFAP. Bottom, A three-dimensional image of multiple BrdU + cells colabeled with NeuN. Arrowhead points to BrdU undifferentiated cell. Units in three-dimensional images; each square side panel of the three-dimensional image is $10 \mu \mathrm{m}$. 
logical conditions but in the context of natural variations in behavior and is modifiable by positive environmental change.

The effect of EC on LRs and not on HRs suggests that some protective experiences may primarily benefit those who are genetically vulnerable to begin with. Although both bred HRs and LRs showed an impact of EC on performance in the plus maze, the effect was more clear-cut in LRs. The contrast between the two lines was more remarkable in the LDB, wherein HRs showed no measurable response to EC, and LRs had a very clear response. The "resistance" of HRs is also notable when examining the effect of EC on FGF2 mRNA levels. Although enriching the environment significantly induced hippocampal FGF2 in bred LRs, it had no impact on FGF2 levels in the bred HRs. The modest effect of EC on HR anxiety behavior in the EPM may be attributable to increased expression of other growth factors such as BDNF or vascular endothelial growth factor (Young et al., 1999; Cao et al., 2004). Overall, the enriched environment rescues the vulnerable LRs and renders them HR-like both in terms of FGF2 expression and anxiety behavior.

These observations are echoed by the studies of exogenous FGF2 as a modulator of anxiety behavior. Chronic FGF2 treatment selectively reduces anxiety behavior in bred LRs, rendering them HR-like. Remarkably, HRs are completely unresponsive to FGF2 treatment, although there is no apparent ceiling effect on the behavior-e.g., in the EPM, the percentage open arm entries was at $36.8 \%$ in HRs, and rather than increasing after treatment, it decreased slightly.

That peripherally administered FGF2 would cross the bloodbrain barrier may appear surprising, but pharmacokinetic analyses demonstrate its rapid transport into the brain (Cuevas et al., 1996; Deguchi et al., 2000). There is also circumstantial evidence that peripheral administration has central effects (Wagner et al., 1999). This unusual feature may relate to FGF2's role in controlling blood-brain barrier permeability and the preservation of its tight junctions (Reuss et al., 2003; Bendfeldt et al., 2007). Nevertheless, one cannot preclude the possibility that there are peripheral consequences of FGF2 that may, in turn, lead its central effects. To address this issue, we have directly compared the antidepressant properties of acute FGF2 intracerebroventricularly or peripherally (our unpublished data) and found them to be similar. Coupled with our observations about differences in endogenous FGF2 correlating with anxiety behavior, the weight of the evidence suggests a direct role of administered FGF2 on central receptors to modulate affective behaviors.

Our findings lead us to hypothesize that FGF2 is a common mediator of environmental influences on anxiety. Thus, social defeat which decreases hippocampal FGF2 (Turner et al., 2008a) increases vulnerability to anxiety (Kinsey et al., 2007). Rats who received low maternal care have low levels of hippocampal FGF2 (Bredy et al., 2003) and exhibit high anxiety (Caldji et al., 1998). Most interestingly, treatment with anxiolytic drugs increase FGF2 expression in hippocampus (Gómez-Pinilla et al., 2000; Mallei et al., 2002). We, therefore, propose that FGF2 may be a key integrator of many influences, be they genetic, environmental, or pharmacological, which define the propensity for anxiety behavior, with higher levels of FGF2 being consistently protective.

How are the effects of FGF2 mediated? The differential impact of exogenous FGF2 on anxiety is accompanied by differential effects on adult neurogenesis. FGF2 increased neurogenesis by enhancing new cell survival selectively in the bred LRs, and this was independent of any observed changes in proliferation in either line. Although it is conceivable that FGF2 treatment resulted in increased proliferation in HRs that led to a dilution of BrdU signal, direct measurement of proliferation using Ki67 does not reveal increased proliferation at the time of death. Thus, any FGF2-induced proliferation in the HRs would have to be transient and not sustained throughout the course of treatment.

Given that bred LRs had lower rates of cell survival basally, our results are consonant with work showing that exogenous FGF2 salvages deficits in cell survival (Rai et al., 2007). Conversely, manipulations that decrease FGF2, including social defeat and low maternal care, are associated with decreased cell survival (Bredy et al., 2003; Thomas et al., 2007). Together, our results suggest that lower levels of hippocampal FGF2 in LRs lead to low cell survival, which in turn leads to a decrease in the generation of new neurons and especially astrocytes and appears associated with increased anxiety behavior.

In the adult, FGF2 shows prominent expression in astrocytes throughout the brain, whereas neuronal expression is almost exclusive to hippocampus (Woodward et al., 1992). Although FGF2 is primarily implicated in neurogenesis, it has also been reported to modulate the number of glia in hippocampus (Cheng et al., 2002). This is consistent with our findings showing an increase in both glial genesis and neurogenesis. It is worth noting that FGF2 caused a fourfold increase in glial genesis compared with a twofold increase in neurogenesis. An important caution is that this increase may be due, at least in part, to an FGF2-induced increase in the expression of GFAP in astrocytes. Thus, cells previously labeled neither especially in LRs, may in fact be astrocytes with low GFAP expression levels attributable to the endogenously low levels of FGF2, and treatment with this factor may induce GFAP expression sufficiently to allow their identification.

Although we are not aware of work linking FGF2 to astrocyte differentiation in adult hippocampus, FGF2 modulates GFAP expression in cortex (Reuss et al., 2003) and induces chromatin remodeling which regulates the ability of cilliary neurotrophic factor to promote astrocyte differentiation in the developing cortex (Song and Ghosh, 2004). Consistent with lower FGF2 basal expression, LR rats show a smaller number of BrdU+ cells that can be classified as glial astrocytes, and, once again, FGF2 treatment rendered them more similar to HRs.

In conclusion, we propose that FGF2 decreases anxiety behavior by increasing the rate of cell survival and promoting the generation of new neurons and especially astrocytes. We also propose that inborn differences in FGF2 levels impact this pattern of differentiation and are associated with differences in anxiety behavior. Overall, higher levels of FGF2 provide resilience to anxiety, and this can be achieved either via genetic endowment or via an environmental manipulation such as environmental complexity, which induces FGF2 and rescues the neural and behavioral phenotype of vulnerable animals. Thus, FGF2 represents an integrator of anxiety behavior and a novel treatment target for the treatment of clinical anxiety and mood disorders.

\section{References}

Akil H, Evans SJ, Turner CA, Perez J, Myers RM, Bunney WE, Jones EG, Watson SJ; Pritzker Consortium (2008) The fibroblast growth factor family and mood disorders. Novartis Found Symp 289:94-96; discussion 97-100, 193-105.

Belluardo N, Wu G, Mudo G, Hansson AC, Pettersson R, Fuxe K (1997) Comparative localization of fibroblast growth factor receptor-1, -2 , and -3 mRNAs in the rat brain: in situ hybridization analysis. J Comp Neurol 379:226-246

Benaroya-Milshtein N, Hollander N, Apter A, Kukulansky T, Raz N, Wilf A, Yaniv I, Pick CG (2004) Environmental enrichment in mice decreases anxiety, attenuates stress responses and enhances natural killer cell activity. Eur J Neurosci 20:1341-1347.

Bendfeldt K, Radojevic V, Kapfhammer J, Nitsch C (2007) Basic fibroblast 
growth factor modulates density of blood vessels and preserves tight junctions in organotypic cortical cultures of mice: a new in vitro model of the blood-brain barrier. J Neurosci 27:3260-3267.

Bredy TW, Grant RJ, Champagne DL, Meaney MJ (2003) Maternal care influences neuronal survival in the hippocampus of the rat. Eur J Neurosci 18:2903-2909.

Caldji C, Tannenbaum B, Sharma S, Francis D, Plotsky PM, Meaney MJ (1998) Maternal care during infancy regulates the development of neural systems mediating the expression of fearfulness in the rat. Proc Natl Acad Sci U S A 95:5335-5340.

Cao L, Jiao X, Zuzga DS, Liu Y, Fong DM, Young D, During MJ (2004) VEGF links hippocampal activity with neurogenesis, learning and memory. Nat Genet 36:827-835.

Cheng Y, Black IB, DiCicco-Bloom E (2002) Hippocampal granule neuron production and population size are regulated by levels of bFGF. Eur J Neurosci 15:3-12.

Clinton S, Miller S, Watson SJ, Akil H (2008) Prenatal stress does not alter innate novelty-seeking behavioral traits, but differentially affects individual differences in neuroendocrine stress responsivity. Psychoneuroendocrinology 33:162-177.

Clinton SM, Vázquez DM, Kabbaj M, Kabbaj MH, Watson SJ, Akil H (2007) Individual differences in novelty-seeking and emotional reactivity correlate with variation in maternal behavior. Horm Behav 51:655-664.

Cuevas P, Fernández-Ayerdi A, Carceller F, Colin S, Mascarelli F, MuñozWillery I, Giménez-Gallego G (1996) Central nervous system distribution of fibroblast growth factor injected into the blood stream. Neurol Res $18: 267-272$.

Davis BA, Clinton SM, Akil H, Becker JB (2008) The effects of noveltyseeking phenotypes and sex differences on acquisition of cocaine selfadministration in selectively bred high-responder and low-responder rats. Pharmacol Biochem Behav 90:331-338.

Deguchi Y, Naito T, Yuge T, Furukawa A, Yamada S, Pardridge WM, Kimura R (2000) Blood-brain barrier transport of 125I-labeled basic fibroblast growth factor. Pharm Res 17:63-69.

Duman RS, Monteggia LM (2006) A neurotrophic model for stress-related mood disorders. Biol Psychiatry 59:1116-1127.

Eckenstein FP (1994) Fibroblast growth factors in the nervous system. J Neurobiol 25:1467-1480.

Evans SJ, Choudary PV, Neal CR, Li JZ, Vawter MP, Tomita H, Lopez JF, Thompson RC, Meng F, Stead JD, Walsh DM, Myers RM, Bunney WE, Watson SJ, Jones EG, Akil H (2004) Dysregulation of the fibroblast growth factor system in major depression. Proc Natl Acad Sci U S A 101:15506-15511.

Flagel SB, Akil H, Robinson TE (2008) Individual differences in the attribution of incentive salience to reward-related cues: implications for addiction. Neuropharmacology 56 [Suppl 1]:139-148.

Gaughran F, Payne J, Sedgwick PM, Cotter D, Berry M (2006) Hippocampal FGF-2 and FGFR1 mRNA expression in major depression, schizophrenia and bipolar disorder. Brain Res Bull 70:221-227.

Gómez-Pinilla F, Dao L, Choi J, Ryba EA (2000) Diazepam induces FGF-2 mRNA in the hippocampus and striatum. Brain Res Bull 53:283-289.

Gorwood P (2004) Generalized anxiety disorder and major depressive disorder comorbidity: an example of genetic pleiotropy? Eur Psychiatry 19:27-33.

Kabbaj M, Devine DP, Savage VR, Akil H (2000) Neurobiological correlates of individual differences in novelty-seeking behavior in the rat: differential expression of stress-related molecules. J Neurosci 20:6983-6988.

Kinsey SG, Bailey MT, Sheridan JF, Padgett DA, Avitsur R (2007) Repeated social defeat causes increased anxiety-like behavior and alters splenocyte function in C57BL/6 and CD-1 mice. Brain Behav Immun 21:458-466.

Malberg JE, Duman RS (2003) Cell proliferation in adult hippocampus is decreased by inescapable stress: reversal by fluoxetine treatment. Neuropsychopharmacology 28:1562-1571.

Malberg JE, Eisch AJ, Nestler EJ, Duman RS (2000) Chronic antidepressant treatment increases neurogenesis in adult rat hippocampus. J Neurosci 20:9104-9110.

Mallei A, Shi B, Mocchetti I (2002) Antidepressant treatments induce the expression of basic fibroblast growth factor in cortical and hippocampal neurons. Mol Pharmacol 61:1017-1024.

Martinowich K, Manji H, Lu B (2007) New insights into BDNF function in depression and anxiety. Nat Neurosci 10:1089-1093.

Ornitz DM (2000) FGFs, heparan sulfate and FGFRs: complex interactions essential for development. Bioessays 22:108-112.

Palmer TD, Ray J, Gage FH (1995) FGF-2-responsive neuronal progenitors reside in proliferative and quiescent regions of the adult rodent brain. Mol Cell Neurosci 6:474-486.

Paxinos G, Watson C (2007) The rat brain in stereotactic coordinates, Ed 6. London: Academic.

Piazza PV, Deminière JM, Le Moal M, Simon H (1989) Factors that predict individual vulnerability to amphetamine self-administration. Science 245:1511-1513.

Pieper AA, Wu X, Han TW, Estill SJ, Dang Q, Wu LC, Reece-Fincanon S, Dudley CA, Richardson JA, Brat DJ, McKnight SL (2005) The neuronal PAS domain protein 3 transcription factor controls FGF-mediated adult hippocampal neurogenesis in mice. Proc Natl Acad Sci USA 102:14052-14057.

Raballo R, Rhee J, Lyn-Cook R, Leckman JF, Schwartz ML, Vaccarino FM (2000) Basic fibroblast growth factor (Fgf2) is necessary for cell proliferation and neurogenesis in the developing cerebral cortex. J Neurosci 20:5012-5023.

Rai KS, Hattiangady B, Shetty AK (2007) Enhanced production and dendritic growth of new dentate granule cells in the middle-aged hippocampus following intracerebroventricular FGF-2 infusions. Eur J Neurosci 26:1765-1779.

Reuss B, von Bohlen und Halbach O (2003) Fibroblast growth factors and their receptors in the central nervous system. Cell Tissue Res 313:139-157.

Reuss B, Dono R, Unsicker K (2003) Functions of fibroblast growth factor (FGF)-2 and FGF-5 in astroglial differentiation and blood-brain barrier permeability: evidence from mouse mutants. J Neurosci 23:6404-6412.

Santarelli L, Saxe M, Gross C, Surget A, Battaglia F, Dulawa S, Weisstaub N, Lee J, Duman R, Arancio O, Belzung C, Hen R (2003) Requirement of hippocampal neurogenesis for the behavioral effects of antidepressants. Science 301:805-809.

Song MR, Ghosh A (2004) FGF2-induced chromatin remodeling regulates CNTF-mediated gene expression and astrocyte differentiation. Nat Neurosci 7:229-235.

Stead JD, Clinton S, Neal C, Schneider J, Jama A, Miller S, Vazquez DM, Watson SJ, Akil H (2006) Selective breeding for divergence in noveltyseeking traits: heritability and enrichment in spontaneous anxiety-related behaviors. Behav Genet 36:697-712.

Thomas RM, Hotsenpiller G, Peterson DA (2007) Acute psychosocial stress reduces cell survival in adult hippocampal neurogenesis without altering proliferation. J Neurosci 27:2734-2743.

Turner CA, Akil H, Watson SJ, Evans SJ (2006) The fibroblast growth factor system and mood disorders. Biol Psychiatry 59:1128-1135.

Turner CA, Calvo N, Frost DO, Akil H, Watson SJ (2008a) The fibroblast growth factor system is downregulated following social defeat. Neurosci Lett 430:147-150.

Turner CA, Gula EL, Taylor LP, Watson SJ, Akil H (2008b) Antidepressantlike effects of intracerebroventricular FGF2 in rats. Brain Res 1224:63-68.

Wagner JP, Black IB, DiCicco-Bloom E (1999) Stimulation of neonatal and adult brain neurogenesis by subcutaneous injection of basic fibroblast growth factor. J Neurosci 19:6006-6016.

Warner-Schmidt JL, Duman RS (2007) VEGF is an essential mediator of the neurogenic and behavioral actions of antidepressants. Proc Natl Acad Sci U S A 104:4647-4652.

Woodward WR, Nishi R, Meshul CK, Williams TE, Coulombe M, Eckenstein FP (1992) Nuclear and cytoplasmic localization of basic fibroblast growth factor in astrocytes and CA2 hippocampal neurons. J Neurosci 12:142-152.

Young D, Lawlor PA, Leone P, Dragunow M, During MJ (1999) Environmental enrichment inhibits spontaneous apoptosis, prevents seizures and is neuroprotective. Nat Med 5:448-453. 